\title{
The Shapley Transfer Procedure for NTU-Games
}

Citation for published version (APA):

Otten, G-J., \& Peters, H. J. M. (2002). The Shapley Transfer Procedure for NTU-Games. In P. Borm, \& HJM. Peters (Eds.), Chapters in Game theory: in Honor of Stef Tijs, Theory and Decision Library Series (pp. 183-203). Kluwer Academic Publishers. https://doi.org/10.1007/0-306-47526-X_9

Document status and date:

Published: 01/01/2002

DOI:

10.1007/0-306-47526-X_9

Document Version:

Publisher's PDF, also known as Version of record

\section{Please check the document version of this publication:}

- A submitted manuscript is the version of the article upon submission and before peer-review. There can be important differences between the submitted version and the official published version of record.

People interested in the research are advised to contact the author for the final version of the publication, or visit the DOI to the publisher's website.

- The final author version and the galley proof are versions of the publication after peer review.

- The final published version features the final layout of the paper including the volume, issue and page numbers.

Link to publication

\footnotetext{
General rights rights.

- You may freely distribute the URL identifying the publication in the public portal. please follow below link for the End User Agreement:

www.umlib.nl/taverne-license

Take down policy

If you believe that this document breaches copyright please contact us at:

repository@maastrichtuniversity.nl

providing details and we will investigate your claim.
}

Copyright and moral rights for the publications made accessible in the public portal are retained by the authors and/or other copyright owners and it is a condition of accessing publications that users recognise and abide by the legal requirements associated with these

- Users may download and print one copy of any publication from the public portal for the purpose of private study or research.

- You may not further distribute the material or use it for any profit-making activity or commercial gain

If the publication is distributed under the terms of Article $25 \mathrm{fa}$ of the Dutch Copyright Act, indicated by the "Taverne" license above, 


\section{Chapter 9}

\section{The Shapley Transfer Procedure for NTU-Games}

by Gert-Jan Otten And Hans Peters

\subsection{Introduction}

A cooperative game is described by sets of feasible utility vectors, one set for each coalition. Such a game may arise from each situation where involved parties can achieve gains from cooperation. Examples range involved parties can achieve gain from from exchange economies to cost allocation between divisions of multinationals or power distribution within political systems. The two centra questions are: which coalitions will form; and on which payoffs will each formed coalition agree. Since an answer to the latter question seems a prerequisite to study the former question of coalition formation, most of the literature has concentrated on the question of payoff distribution. Specifically, the usual assumption is that the grand coalition of all players will form and then the question is which payoff vector(s) this coalition will agree upon.

This question has been studied extensively for two special cases:
(a) Tames with transferable utility, and pure bargaining games.

In a game with transferable utility, what each coalition can do is described by just one number: the total utility or payoff, which that coalition can distribute among its members in any way it wants. The underlying assumption is the presence of a common medium of exchange in which the players' utilities are linear. For instance, the payoff is in monetary units and the players have linear utility for money.

183

P. Borm and H. Peters (eds.), Chapters in Game Theory, 183-203.
O2002 Kluwer Academic Publishers. Printed in the Netherlands. 
In a pure bargaining game intermediate coalitions-coalitions other than the grand coalition or individual players-play no role. Because of these simplifying features, both types of games are easier to analyse than general cooperative games, also called games with nontransferable utility or NTU-games.

A solution is a map that assigns to every game within a certain subclass of NTU-games a feasible payoff vector or set of feasible payoff vectors for the grand coalition. In an important article Shapley (1069) proposed a procedure to extend single class of games with tran conditions to conditions, to NTU-games. This procedure works as follows. For a given For evame consider any vector of nonnegative weights for the players. For every coalition, maximize the correspondingly weighted sum of the utilities of its members over the set of feasible payoffs of that coalition. Regard these coalitional maxima as a game with transferable utility and apply the given solution for transferable utility games to this game: those payoff vectors of the original NTU-game that when similarly werght belong to the solution of the TU-game, are defined to be in weighted, of the NTU-game. The complete solution of the NTU the solution obtained by repeating this procedure for every possbu-game is then

This Shapley transfer procedure has been applied in particular to the Shapley value for games with transferable utility (Shapley, 1953) resulting in the 'nontransferable utility value' (Aumann, 1985) for NTUgames. For pure bargaining games, this solution coincides with bargaining solution, proposed by Nash (1950) for the case of twe Nash As indicated, however, by Shapley (1969) the procedure of two players. to a variety of solutions; also the (1969) the procedure can be applied holds under quite mild ; also the existence result established by Shapley NTU $\tau$-val quite $\tau$-value studied in Borm et al. (1992). Another example is the so called 'Viner core' studied by Qin (1994) but proposed earlier by Shapley

The main objective of the present contribution is twofold. First we review and extend the Shapley transfer procedure; the extension is to any compact and convex valued, continuous solution. Shapley's existence result will be re-established for this extension. Second, solutions that are obtained by this procedure. Second, we characterize be seen as an alternative by this procedure. This characterization can The seen as an alternative description of the Shapley transfer procedure. The price for obtaining existence and characterization within the same ramework is the occurrence of zero weight
Section 2 introduces the Shapley transfer procedure. Section 3 contains the existence result and a digression on a well known and earlier procedure proposed by Harsanyi $(1959,1963)$ - to which the existence result applies equally. In Section 4 the announced characterization is presented Section 5 discusses applications to several TU solutions the Shapley value, the core, the nucleolus, and the $\tau$-value. Sections: the cludes.

Notations. For a finite subset $S$ of the natural numbers let $\mathbb{R}_{+}^{S}$ denote the nonnegative orthant of $\mathbb{R}^{S}$. For $x, y \in \mathbb{R}^{S}$ denote $x>y$ if $x_{i}>y_{i}$ for every $i \in S$, and denote $x \geq y$ if $x_{i} \geq y_{i}$ for every $i \in S$. The vector inequalities $<, \leq$ are defined analogously. The $\cdot$ denotes the usual inner product: $x \cdot y=\sum_{i \in S} x_{i} y_{i}$. The product $x y$ denotes the vector in $\mathbb{R}^{S}$ with $i$ th coordinate equal to $x_{i} y_{i}(i \in S)$. For $Y \subseteq \mathbb{R}^{S}, x Y:=\{x y$ $y \in Y\}$; for a real number $a, a Y:=(a, \ldots a) Y$ For another finite set of natural numbers $M$ with $M \cap S \neq \emptyset, x \in \mathbb{R}^{S}$ and $Y \subseteq \mathbb{R}^{S}$ let $x_{M} \in \mathbb{R}^{M \cap S}$ be defind by $\left(x_{M}\right)_{i}=x_{1}$ for every $i \in M \cap S ;$ let $Y_{M}:=\left\{\left(x_{M} \in \mathbb{R}^{M \cap S}: y \in Y\right\}\right.$. Thus, the set $Y_{M}$ is the pojection $Y$ $Y_{M}:=\left\{y_{M} \in \mathbb{R}{ }^{M} S: y \in Y\right\}$. Thus, the set $Y_{M}$ is the projection of $Y$ on the $M \cap S$-plane.

\subsection{Main Concepts}

Let $N=\{1,2, \ldots, n\}$ denote the set of players. A coalition is a nonempty subset of $N$. A subset $D \subseteq \mathbb{R}_{+}^{S}$ is comprehensive if $x \in D$ and $y \leq x$ imply $y \in D$ for all $x, y \in \mathbb{R}_{+}^{S}$. The Pareto optimal subset of $D$ is the set

$P O(D):=\{x \in D:$ for all $y \in D$, if $x \leq y$ then $x=y\}$

and the weakly Pareto optimal subset of $D$ is the set

$W P O(D):=\left\{x \in D:\right.$ for all $y \in \mathbb{R}_{+}^{S}$, if $x<y$ then $\left.y \notin D\right\}$.

A nontransferable utility game or NTU-game is a pair $(N, V)$ where $V$ assigns to every coalition $S$ a feasible set $V(S)$ such that

(N1) $V(\{i\})=\{0\} \subseteq \mathbb{R}$ for every $i \in N$;

(N2) $V(S)$ is a nonempty compact convex and comprehensive subset of $\mathbb{R}_{+}^{S}$ for every coalition $S$;

(N3) $P O(V(S))=W P O(V(S))$ for every coalition $S$. 
These assumptions, though restrictive, are still quite standard. The normalization in (N1) is mainly for convenience; it is not innocent because together with (N2) it implies, for instance, that every coalition can at least as good as singleton coalitions. The convexity assumption in (N2) may arise from the players having von Neumann-Morgenstern utility functions over uncertain outcomes, or concave ordinal utility function over bundles of goods. It is essential to what follows. Condition (N3) means that, for every coalition, every weakly Pareto optimal point is also Pareto optimal: there are no flat segments in the weally Pareto optimal boundary of $V(S)$. One consequence is that if $x \in P O(V(S))$ with $x_{i}>0$ for some $i \in S$, then there is a $y \in P O\left(V(S)\right.$ with $0<y_{i}<x_{i}$ and $y_{j}>x_{j}$ for all $j \neq i$; note that in that case $y>0$. It follo $y_{i}$ particular, that either $V(S)=\{0\}$ or the is a $y \in V(S)$. It follows, in

If $V(S)=\{0\} \subset R^{S}$ for $\{0\}$ or there is a $y \in V(S)$ with $y>0$. pure bargaining $\subseteq \mathbb{R}_{+}^{S}$ for every $S \neq N$, then $(N, V)$ is called a pure bargaining game. If for every coalition $S$ there is a nonnegative $(N, V)$ ser $v(S)$ such that $V(S)=\left\{x \in \mathbb{R}_{+}^{S}: \sum_{i \in S} x_{i} \leq v(S)\right\}$, then $(N, V)$ is called a game with transferable utility or TU-game. Such a TU. game is sometimes also denoted by $(N, v)$. Our definition deviates from the usual one in that all payoff vectors are restricted to the notes from orthant.

Instead of $(N, V)$ or $(N, v)$ we will usually write $V$ or $v$, with the understanding that the player set is $N$.

The class of NTU-games [TU-games, pure bargaining games] with player set $N$ is denoted by $\mathcal{G}^{N}\left[\mathcal{T}^{N}, \mathcal{B}^{N}\right]$. Often the superscript ' $N$ ' is omitted. Subclasses are denoted by $\mathcal{G}^{\prime}, \mathcal{T}^{\prime}$, etc.

Let $\mathcal{G}^{\prime} \subseteq \mathcal{G}$ be a subclass of NTU-games. An NTU-solution is a correspondence $\Phi: \mathcal{G}^{\prime} \rightarrow \mathbb{R}_{+}^{N}$ that assigns to each NTU-game $V \in G^{\prime}$ set $\Phi(V) \subseteq V(N)$. (We use $\rightarrow$ to denote a correspondence, i. a setvalued function.) If $\mathcal{G}^{\prime} \subseteq \mathcal{T}$ then $\Phi$ is also called a TU-solution. Usully

TU-solutions are denoted by small characters, e $\varphi$. A TU-solution $\varphi$ defined on $\mathcal{T}^{\prime} \subseteq \mathcal{T}$, $\varphi$.

the following three conditions. $V$ and a real number $a>0$. $(a V)(S):=a V(S)$ for every we denote by $a V$ the NTU-game with y coalition $S$ (T1) $\varphi(V)$ is a nonempty, compact, and convex subset of $P O(V(N))$
for every $V \in \mathcal{T}^{\prime}$;

(T2) $\varphi$ is continuous on $T^{\prime}$

(T3) $\varphi$ is homogeneous, that is, for every $V \in \mathcal{T}^{\prime}$ and real number $a>0$

$a V \in \mathcal{T}^{\prime}$ implies $\varphi(a V)=a \varphi(V)$.

Here, continuity is meant with respect to the restriction to $\mathcal{T}^{\prime}$ of the $\mathrm{Eu}-$

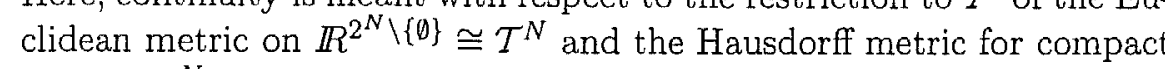
sets in $\mathbb{R}^{N}$. Conditions (T1), (T2) and (T3) are not very restrictive Most known single-valued solutions (e.g., Shapley value, nucleolus, $\tau$ value) are continuous and homogeneous on the classes of TU-games on which they are defined. The best known multi-valued concept, the core satisfies (T1), (T2), and (T3) on the class of balanced games. See Section 5 for some of the details.

For an arbitrary NTU-game $V$ and an arbitrary vector $\lambda \in \mathbb{R}_{+}^{N} \backslash\{0\}$ the associated $\lambda$-transfer game $V^{\lambda}$ is the transferable utility game defined by

$V^{\lambda}(S):=\left\{x \in \mathbb{R}_{+}^{S}: \sum_{i \in S} x_{i} \leq v^{\lambda}(S)\right\}$

where

$v^{\lambda}(S):=\max \left\{\lambda_{S} \cdot x: x \in V(S)\right\}$

for every coalition $S$. By (N1) and (N2) these numbers $v^{\lambda}(S)$ are well defined. For a class $\mathcal{G}^{\prime}$ of NTU-games denote by

$T\left(\mathcal{G}^{\prime}\right):=\left\{V^{\lambda} \in \mathcal{T}^{N}: V \in \mathcal{G}^{\prime}, \lambda \in \mathbb{R}_{+}^{N} \backslash\{0\}\right\}$

the class of all TU-games that arise as transfer games associated with NTU-games in $\mathcal{G}^{\prime}$. Let $\varphi: \mathcal{T}^{\prime} \rightarrow \mathbb{R}^{N}$ be a regular TU-solution defined on $T\left(\mathcal{G}^{\prime}\right)$. We extend $\varphi$ to an NTU-solution $\bar{\varphi}: \mathcal{G}^{\prime} \rightarrow \mathbb{R}^{N}$, as follows. For each $V \in \mathcal{G}^{\prime}$ and $x \in P O(V(N))$,

$x \in \bar{\varphi}(V): \Leftrightarrow \exists \lambda \in \mathbb{R}_{+}^{N} \backslash\{0\}, y \in \varphi\left(V^{\lambda}\right): y=\lambda x$

One way to understand this procedure (cf. Qin, 1994) is to think of the players as countries and of the coordinates of $\lambda$ as exchange rates between these countries. Then the transfer game $V^{\lambda}$ expresses what coalitions of countries can do in real monetary terms, and the vector $y$ represents a payoff distribution in real monetary terms. If this payoff distribution is feasible in terms of the original individual currencies, then it is a solution of the game.

We call $\bar{\varphi}$ the transfer solution associated with $\varphi$. Observe that not all of the properties in (T1) and (T2) are trivially inherited by $\bar{\varphi}$. In this contribution we will be mainly concerned with existence, i.e., nonemptiness. 
The transfer procedure can also be applied to TU-games, resulting ution to the associated transfer solution on

Lemma 9.1 Let $\varphi$ be a regular $T U$-solution on a class $\mathcal{T}^{\prime}$. Let $V \in \mathcal{T}^{\prime}$ with $T(\{V\}) \subseteq T^{\prime}$. Then $\varphi(V) \subseteq \bar{\varphi}(V)$

Proof. Let $x \in \varphi(V)$ and $\lambda=(1,1, \ldots, 1)$. Then $V^{\lambda}(S)=V(S)$ for every coalition $S$, so $\lambda x=x \in \varphi(V)=\varphi\left(V^{\lambda}\right)$. Hence, $x \in \bar{\varphi}(V)$. for

Observe that actually we do not need regularity for Iemma 0.1 to botd. The inclusion in the lemma, solutions, as the following example shows, can be strict, even for regular

Example 9.2 Let $N=\{1,2,3\}$ and for every $v \in \mathcal{T}^{N}$ let

$\epsilon(v):=\frac{1}{3} \min \{v(N) / 2 v(\{2,3\}), 1\}$ if $v(\{2,3\})>0$ and $\epsilon(v):=\frac{1}{3}$ oth erwise. Define $\varphi: \mathcal{T}^{N} \rightarrow \mathbb{R}^{N}$ by $\varphi(v):=\left\{\left(\frac{1}{3}+2 \epsilon(v), \frac{1}{3}-\epsilon(v), \frac{1}{3}-\right.\right.$ $\epsilon(v)) v(N)\}$ for every $v \in \mathcal{T}^{N}$. Observe that $\varphi$ is a regular TU-solution. Consider the game $w$ with $w(S)=1$ for all coalitions $S$ with more than one player. Then $\epsilon(w)=\frac{1}{6}$ and $\varphi(w)=\left\{\left(\frac{2}{3}, \frac{1}{6}, \frac{1}{6}\right)\right\}$. Let $\lambda=(4,1,1)$. Then $w^{\lambda}(N)=4, w^{\lambda}(\{2,3\})=1, \epsilon\left(w^{\lambda}\right)=\frac{1}{3}$, and $\varphi\left(w^{\lambda}\right)=\{(4,0,0)\}$ Since $\lambda(1,0,0)=(4,0,0)$, it follows that $(1,0,0) \in \bar{\varphi}(w)$. is strictly larger than $\varphi(w)$.

Note that the possibility of the strict inclusion $\varphi(w) \subset \bar{\varphi}(w)$ is not due to the possibility of zero coordinates of $\lambda$, but to the occurrence of boundary solution points with zero coordinates.

In Section 5 we present examples showing that also for TU-solutions such as the core and the nucleolus the inclusion in Lemma 9.1 can strict: hence, the transfer procedure applied to TU-games may add be ditional solution outcomes. This will not be the case for the Shapley value or the $\tau$-value

We conclude this section by reviewing a well known application of thi procedure. Let, as above, $\mathcal{B}=\mathcal{B}^{N}$ be the class of pure bargaining games The class $T(\mathcal{B})$ of associated transfer games consists of all TU- games with $v(S)=0$ for all $S \neq N$. Consider the equal of all TU-games $v$ $T(\mathcal{B})$, that is,

$\varepsilon(v):=\left\{\frac{1}{n}(v(N), \ldots, v(N))\right\}$ for every $v \in T(\mathcal{B})$
Let $V$ be a pure bargaining game such that $x \in V(N)$ for some $x>0$ This is without loss of generality since the only alternative case is $V(N)=\{0\}$, see above.) Then $v^{\lambda}(N)>0$ for every $\lambda \in \mathbb{R}^{N} \backslash\{0\}$. $z \in P O(V(N))$, then $z \in \bar{z}(V)$ if and only if there is a $\lambda \in \mathbb{R}^{N} \backslash\{0\}$ such that $\lambda z=\left(v^{\lambda}(N) / n\right)(1, \ldots, 1)$. Since $v^{\lambda}(N)>0$ for $\mathbb{R}_{+} \backslash\{0\}$ follows that both $z$ and $\lambda$ are positive follows that both $z$ and $\lambda$ are positive and hence $\lambda=k\left(1 / z_{1}, \ldots, 1 / z_{n}\right)$, where $k=v^{\lambda}(N) / n$, and by definition of $v^{\lambda}(N)$, there is a hyperplane supporting $V(N)$ at $z$ with normal $\lambda$. Consider the product $\prod_{i \in N} x_{i}$ on $V(N)$. At $z$, the supporting hyperplane to the level curve of this product has normal $\left(1 / z_{1}, \ldots, 1 / z_{n}\right)$ as follows straightforwardly by partial differentiation; hence this hyperplane also supports $V(N)$. It follows that the product $\prod_{i \in N} x_{i}$ is maximized on $V(N)$ at $z$. So $z$ is the $(n-$ person) Nash bargaining solution outcome for $V$ (Nash, 1950). Thus, $\bar{\varepsilon}(V)$ contains exactly one point, which is the Nash bargaing . Thus,

\subsection{Nonemptiness of Transfer Solutions}

In this section we show that under the imposed conditions a transfer solution assigns a nonempty set of payoff vectors to any NTU-game for which it is defined. This result extends Shapley's (1969) existence for which it is defned. This result extends Shapley's (1969) existence result to the case where the $T U$-solution under consideration may be a correspondence. The proof closely follows Shapley's proof.

In order to obtain a compact set we normalize the $\lambda$-transfer vectors. Specifically, let $\Delta^{N}:=\left\{\lambda \in \mathbb{R}_{+}^{N}: \sum_{i \in N} \lambda_{i}=1\right\}$ denote the $(n-1)$ dimensional simplex in $\mathbb{R}^{N}$. Elements of $\Delta^{N}$ are also called weight vectors.

Theorem 9.3 Let $V \in \mathcal{G}^{N}$ and let $\mathcal{T}^{\prime}:=\left\{V^{\lambda}: \lambda \in \Delta^{N}\right\}$

Let $\varphi: \mathcal{T}^{\prime} \rightarrow \mathbb{R}^{N}$ be a regular $T U$-solution. Then $\bar{\varphi}(V) \neq \emptyset$.

Proof. Define the correspondence $P: \Delta^{N} \rightarrow \mathbb{R}^{N}$ by

$P(\lambda):=\left\{\pi \in \mathbb{R}^{N}: \sum_{i \in N} \pi_{i}=0, \exists x \in \lambda P O(V(N)), y \in \varphi\left(V^{\lambda}\right)[\pi=y-x]\right\}$

So $P$ assigns to a weight vector the set of all 'sidepayments' by which solution payoff vectors of the transfer game are carried over to feasible elements of the correspondingly weighted NTU-game. Note that $P$ is nonempty, convex and compact valued. Moreover, it is upper semicontinuous since $\lambda \mapsto \varphi\left(V^{\lambda}\right)$ is continuous in the Hausdorff metric, and 
are inherited by the correspondence semicontinuous. These properties $L: \lambda \mapsto \lambda+P(\lambda):=\{\lambda+\pi: \pi \in P(\lambda)\}$.

$L: \lambda \mapsto \lambda+P(\lambda):=\{\lambda+\pi: \pi \in P(\lambda)\}$

In particular this implies compactness of the set $\bigcup_{\lambda \in \Delta^{N}} L(\lambda)$. Therefore we can find a compact and convex subset $D$ of $\left\{x \in \mathbb{R}^{N}: \sum_{i \in N} x_{i}=1\right\}$ such that $D \supseteq \Delta^{N} \cup\left(\cup_{\lambda \in \Delta^{N}} L(\lambda)\right)$. Extend the correspondence $L$ to $D$
by defining, for every $d \in D$

$L(d):=L(f(d))$

where $f_{i}(d):=\max \left\{0, d_{i}\right\} / \sum_{j \in N} \max \left\{0, d_{j}\right\}$ for every $i \in N$ Since the projection $f$ is also continuous, Kakutani's fixed point theorem If $d^{*}=\lambda^{*}$, then $0 \in P\left(\lambda^{*}\right)$, hence by definition of $P$ there exists
\[ \varphi\left(V^{\lambda^{*}}\right) \cap \lambda^{*} P O(V(N)) \text {. So } y \in \bar{\varphi}(V) \text {. } \] $y \in \varphi\left(V^{\lambda^{*}}\right) \cap \lambda^{*} P O(V(N))$. So $y \in \bar{\varphi}(V)$ and the proof is complete. Suppose $d^{*} \neq \lambda^{*}$. We will show that this is not possible. In this case $L\left(\lambda^{*}\right)=\lambda^{*}+P\left(\lambda^{*}\right)$, there exists $d_{i}^{*}$. Since $d^{*} \in L\left(d^{*}\right)=L\left(f\left(d^{*}\right)\right)=$ by definition we have $x \geq 0$ for all $x^{*}-\pi^{*} \in \lambda^{*} P O(V(N))$ the for all $x \in \varphi\left(V^{\lambda^{*}}\right)$; take $x^{*} \in \varphi\left(V^{\lambda^{*}}\right)$ with

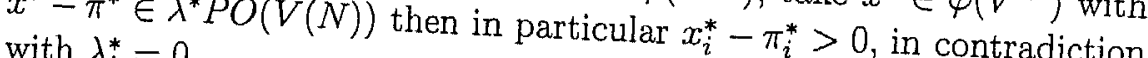

By checking the proof of this theorem one observes that it would be valid for any other transfer procedure as long as the resulting TUld be for a specific NTU-game depend continuously on the woigh TU-games example of such a procedure is the one underlying weight vector. On Harsanyi NTU-value (Harsanyi, 1959, 1963). In order to define this proceduse

a weight vector. We first assume that $\lambda$ be an NTU-game and let $\lambda$ be We recursively define the dividends $\lambda$ has only positive coordinates. $d_{\{i\}}:=0$ for every $i \in N$, and for $S$ 作 with more than one player:

$=\max \left\{t \in \mathbb{R}: x^{S}(t) \in V(S)\right\}$

where $x_{i}^{S}(t)$ for every $i \in S$ is given by

$x_{i}^{S}(t)=\lambda_{i}^{-1}\left(t+\sum_{T: i \in T \subseteq S, T \neq S} d_{T}\right)$

Observe that $x_{i}^{\{i, j\}}(t)=t / \lambda_{i}$ for all $i, j \in N$, so that $d_{[i, j)}\left(\lambda_{j}^{-1}, \lambda_{j}^{-1}\right)$ is the maximal element in the direction reciprocal to $\lambda_{\{i, j\}}$ on the boundary of $V(\{i, j\})$. For coalitions of three and more players the idea is similar but the starting point is, generally speaking, no longer the origin. Now define, for each coalition $S$, the vector $x^{S}:=x^{S}\left(d_{S}\right)$, and let $v_{d}^{\lambda}$ be defined by

$v_{d}^{\lambda}(S):= \begin{cases}\lambda_{S} \cdot x^{S} & \text { if } S \neq N \\ \max \{\lambda \cdot x: x \in V(N)\} & \text { if } S=N\end{cases}$

Note that there is an asymmetry in this definition between the grand coalition and the smaller coalitions. We comment on this below.

That $v_{d}^{\lambda}$ is actually a TU-game follows immediately since $x^{S} \in$ $V(S) \subseteq \mathbb{R}_{+}^{S}$ by definition. For positive $\lambda$ the game $v_{d}^{\lambda}$ depends continuously on $\lambda$. We still have to define the games $v_{d}^{\lambda}$ for the case where $\lambda$ has one or more coordinates equal to zero. The following lemma shows that this can be done by taking limits.

Lemma 9.4 Let $\lambda \in \Delta^{N}$ and let $V$ be an NTU-game. Then there is a TU-game $v$ such that $v_{d}^{\lambda^{k}} \rightarrow v$ for any sequence $\lambda^{1}>0, \lambda^{2}>0, \ldots$ in $\Delta^{N}$ with $\lambda^{k} \rightarrow \lambda$

Proof. Let $\lambda^{1}>0, \lambda^{2}>0, \ldots$ in $\Delta^{N}$ with $\lambda^{k} \rightarrow \lambda$. Then obviously $v_{d}^{\lambda^{k}}(N)$ converges: call the limit $v(N)$. For $S \neq N$ and $k \geq 1$, let $\left(x^{S}\right)^{k}$ be the vector as defined above associated with $\lambda^{k}$. Since $\left(x^{S}\right)^{k}$ is in the compact set $V(S)$ for every $k$, we have $\lim _{k \rightarrow \infty} \lambda_{S}^{k} \cdot\left(x^{S}\right)^{k}=\lim _{k \rightarrow \infty} \lambda_{S^{\prime}}^{k}$. $\left(x^{S}\right)_{S_{S}^{\prime}}^{k}$, where $S^{\prime}:=\left\{i \in S: \lambda_{i}>0\right\}$. Since $\left(x^{S}\right)_{S^{\prime}}^{1},\left(x^{S}\right)_{S^{\prime}}^{2} \ldots$ converges to $x_{S^{\prime}}^{S}$ for some $x^{S} \in V(S)$, the proof is complete by defining $v(S):=$

In view of this lemma we can define a collection of Harsanyi transfer games $V_{d}^{\lambda}$ for every $\lambda \in \Delta^{N}$. The exenter defined ${ }_{d}$ for exterion of a TU-solution can be cefined completely analogous as in the case of the Shapley transfer proon $\lambda$. Since the Harsanyi transfer games again depend continuously on $\lambda$ the existence result, Theorem 9.3, also holds for this case:

Theorem 9.5 Under the assumptions in Theorem 9.3, $\hat{\varphi}(V) \neq \emptyset$, where $\hat{\varphi}$ is the extension of $\varphi$ according to the Harsanyi procedure.

One application is to take the Shapley value as a TU-solution: this results in the so-called Harsanyi NTU-value under the Hasany tranfer procedure. Specifically, if in the definition of the TU would take $v_{d}^{\lambda}(N)=\lambda \cdot x^{N}$, then $x^{N}$ would be the Shapley value of

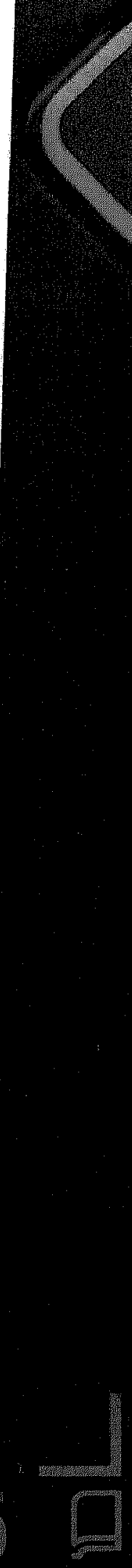


resulting TU-game. If, additionally, $x^{N}$ maximizes $\lambda \cdot x$ on $V(N)$ then $x^{N}$ is said to be in the Harsanyi solution. Hence, such points $x^{N}$ result by applying the Harsanyi transfer procedure to the Shapley TU-solution. See Hart (1985) for a characterization.

\subsection{A Characterization}

In this section we present a general characterization of NTU-solutions that are obtained by extending regular TU-solutions through the Shapy transfer procedure.

Let $\Phi$ be an NTU-solution defined on a class $\mathcal{G}^{\prime}$ of NTU-games. We list the following possible properties of $\Phi$.

Property 9.6 $\Phi$ is Pareto optimal if $\Phi(V) \subseteq P O(V(N))$ for every $V \in$

This property needs no further explanation.

In the following property, for a game $V$ and a positive vector $\alpha \in \mathbb{R}^{N}$ $\alpha V$ denotes the game defined by $(\alpha V)(S):=\alpha_{S} V(S)$ for every nonempty
coalition $S \subseteq N$.

Property 9.7 $\Phi$ is scale covariant if for every $V \in \mathcal{G}^{\prime}$, every $x \in \Phi(V)$, and every $\alpha \in \mathbb{R}^{N}$ with $\alpha>0$ we have: if $\alpha V \in \mathcal{G}^{\prime}$, then $\alpha x \in \Phi(\alpha V)$. One possible interpretation of this property is that the players have cardinal utility functions that are unique only up to a positive affine transformation.

For the next two properties and the ensuing characterization result we need to introduce some additional notation. For a game $V$, a nonempty coalition $S$ and a nonnegative vector $\alpha \in \mathbb{R}^{N}$ denote by $H(V, S, \alpha)$ the halfspace of $\mathbb{R}^{S}$ of all points on or below the hyperplane with normal $\alpha_{S}$ supporting $V(S)$ from above, and by $\partial H(V, S, \alpha)$ its boundary. Thus, for any point of tangency $y \in P O(V(S)) \cap H(V, S, \alpha)$ $H(V, S, \alpha)=\left\{x \in \mathbb{R}^{S}: \alpha_{S} \cdot x \leq \alpha_{S} \cdot y\right\}$

and

$\partial H(V, S, \alpha)=\left\{x \in \mathbb{R}^{S}: \alpha_{S} \cdot x=\alpha_{S} \cdot y\right\}$.

For $\alpha \in \mathbb{R}^{N}$ with $\alpha>0$, an NTU-game $V$ is called an $(\alpha$-) hyperplane game if for every nonempty coalition $S$ the is $P O(V(S))$ colyperplane 作

TU-game is a hyperplane game.
Property 9.8 $\Phi$ is expansion independent if for every $V \in \mathcal{G}^{\prime}$ and $x \in$ $\Phi(V) \cap P O(V(N))$ there is a $\lambda \in \mathbb{R}_{+}^{N} \backslash\{0\}$ with $x \in \partial(H(V, N, \lambda))$ such that, for $L:=\left\{i \in N: \lambda_{i}>0\right\}$ and for all $V^{\prime} \in \mathcal{G}^{\prime}$ satisfying $V(S)_{L} \subseteq V^{\prime}(S)_{L} \subseteq H(V, S, \lambda)$ for alt $S$ with $S \cap L \neq 0$, we have: $x \in \Phi\left(V^{\prime}\right)$.

This property captures the essence of the Shapley transfer procedure. If a game is extended by allowing sidepayments that preserve the utility comparison ratios between the players at a certain solution outcome then that outcome should still belong to the solution of the extended game. The property, naturally, requires this to hold also for game in between the original game and the game extended by sidepayments, although this is not needed for the characterization theorem below. If the utility comparison ratios are not uniquely determined-which is the case if there is no unique supporting hyperplane at the solution outcomehen the requirement applies to at least one set of ration. A proviso is made for the zero conples to af lost one set of ratios. A proviso is made for the zero compon to at in Property 9.8; by property (N3) of an NTU-game it follows that player outside the set $L$ must have zero at $x$, and then Property 9.8 implies that these players will stay at zero in the solution.

The expansion independence property was first introduced by Thomson (1981) in the context of pure bargaining problems.

The fourth property is in a sense the 'dual' of Property 9.8 .

Property $9.9 \Phi$ is contraction independent if for every hyperplane game $V \in \mathcal{G}^{\prime}$, every $x \in \Phi(V)$, and every $V^{\prime} \in \mathcal{G}^{\prime}$ with $x \in V^{\prime}(N)$ such that, for $L:=\left\{i \in N: x_{i}>0\right\}$, the set $P O\left(V(S)_{L}\right)$ supports $V^{\prime}(S)_{L}$ from above for every coalition $S$ with $S \cap L \neq \emptyset$, we have: $x \in \Phi\left(V^{\prime}\right)$.

This property is a variant of the well known 'independence of irrelevant alternatives' condition proposed by Nash (1950).

The characterization result is as follows.

Theorem 9.10 Let $\Phi$ be an NTU-solution defined on a class $\mathcal{G}^{\prime}$ of $N T U$-games containing all hyperplane games, and let $\varphi$ be a regular $T U$-solution on $T\left(\mathcal{G}^{\prime}\right)$. The following two statements are equivalent:

(i) $\Phi$ satisfies Properties 9.6-9.9 and $\Phi(V)=\bar{\varphi}(V)$ for every $V \in$ $T\left(\mathcal{G}^{\prime}\right)$.

(ii) $\Phi(V)=\bar{\varphi}(V)$ for every $V \in \mathcal{G}^{\prime}$. 

Proof. In this proof we use the following fact, the proof of which is

Fact. Let $V \in \mathcal{G}^{\prime}$ and let $\alpha, \lambda \in \mathbb{R}_{+}^{N}$ with $\alpha>0$. Then $(\alpha V)^{\lambda}=V^{\alpha \lambda}$ Proof of $(i i) \Rightarrow(i)$. Assume that (ii) holds. To prove (i), we have to

Pareto optimality of $\bar{\varphi}$ follows by definition.

For scale covariance, let $V \in \mathcal{G}^{\prime}$ and $\alpha \in \mathbb{R}^{N}$ with $\alpha>0$ and
$\alpha V \in \mathcal{G}^{\prime}$ $\alpha V \in \mathbb{R}^{N} \backslash\{0\}$ by $x \in \bar{\varphi}(V)$ and $\mu \in \mathbb{R}_{+}^{N} \backslash\{0\}$ with $\mu x \in \varphi\left(V^{\mu}\right)$. Define $\lambda \in \mathbb{R}_{+} \backslash\{0\}$ by $\lambda_{i}:=\mu_{i} / \alpha_{i}$ for every $i \in N$. Then $\lambda \alpha=\mu$, so $\lambda \alpha x \in$ $\varphi\left(V^{\lambda}\right)=\varphi\left((\alpha V)^{\lambda}\right)$ by Fact (i). Hence, $\alpha x \in \bar{\varphi}(\alpha V)$. This implies scale covariance of $\bar{\varphi}$.

For expansion independence, take $V \in \mathcal{G}^{\prime}$ and $x \in \bar{\varphi}(V)$. Let $\lambda \epsilon$ $\mathbb{R}_{+}^{N} \backslash\{0\}$ with $\lambda x \in \varphi\left(V^{\lambda}\right)$. Then $x \in \partial(H(V, N, \lambda))$. Let $L$ and $V^{\prime}$ as in the definition of Property 9.8. Then by (N3) $x_{N \backslash L}=0,\left(V^{\prime}\right)^{\lambda}=$ $V^{\lambda}$ and $x \in P O\left(V^{\prime}(N)\right)$. Hence, $x \in \bar{\varphi}\left(V^{\prime}\right)$, which proves expansion independence.

Finally, for contraction independence, let $V \in \mathcal{G}^{\prime}$ be an $\alpha$-hyperplane game $(\alpha>0)$. Let $x \in \bar{\varphi}(V)$, hence there is a $\lambda \in \mathbb{R}_{+}^{N} \backslash\{0\}$ with $\lambda x \in \varphi\left(V^{\lambda}\right)$. Observe that for $i \in L:=\left\{j \in N: x_{j}>0\right\}$ we have $\lambda_{i}>0$, otherwise there would be a $y$ in $V(N)$ with $\lambda \cdot y>\lambda \cdot x_{1}$ contrading $\lambda x \in \varphi\left(V^{\lambda}\right)$. Then for $V^{\prime}$ as in Property 9.9 it follows that $\left(V^{\prime}\right)^{\lambda}=V^{\lambda}$. Together with $x \in V^{\prime}(N)$ this implies $x \in \bar{\varphi}\left(V^{\prime}\right)$.

Proof of $(i) \Rightarrow(i i)$. Assume that (i) holds. Let $V \in \mathcal{G}^{\prime}$ and $x \in \Phi(V)$. We prove that $x \in \bar{\varphi}(V)$ and, thus, that $\Phi(V) \subseteq \bar{\varphi}(V)$. By Pareto pptimality and expansion independence of $\Phi$ we can take $\lambda$ and $L$ in Property 9.8. Define $\lambda^{\prime} \in \mathbb{R}^{N}$ by $\lambda_{i}^{\prime}:=\lambda_{i}$ if $i \in L$ and $\lambda_{i}^{\prime}:=1$. $i \in N \backslash L$. For $V^{\prime}$ as in Property 9.8 take a $\lambda^{\prime}$-hyperplane and $\lambda_{i}:=1$ 9.8, expansion independence, implies $x \in \Phi\left(V^{\prime}\right)$. By scale Property $\left(\lambda^{\prime}\right)^{-1} x \in \Phi\left(\left(\lambda^{\prime}\right)^{-1} V^{\prime}\right)$, where $\left(\lambda^{\prime}\right)_{i}^{-1}:=1 / \lambda_{i}^{\prime}$ for every $i$ covariance, $\left(\lambda^{\prime}\right)^{-1} V^{\prime}$ is a TU-game, this implies $\left(\lambda^{\prime}\right)^{-1} x \in \bar{\varphi}\left(\left(\lambda^{\prime}\right)^{-1} V^{\prime}\right)$. Since scale covariance, $x \in \bar{\varphi}\left(V^{\prime}\right)$, and by Property For the converse implication by Property 4: $x \in \bar{\varphi}(V)$

which complo show that $x \in \Phi(V)$, $\varphi\left(V^{\lambda}\right)$ completes the proof of the theorem. Let $\lambda \in \mathbb{R}_{+}^{N} \backslash\{0\}$ with $\lambda x \in$ $\varphi\left(V^{\lambda}\right)$. By Lemma 9.1, $\varphi\left(V^{\lambda}\right) \subseteq \bar{\varphi}\left(V^{\lambda}\right)$; and since $\bar{\varphi}\left(V^{\lambda}\right)=\Phi\left(V^{\lambda}\right)$, we have $\lambda x \in \Phi\left(V^{\lambda}\right)$. Define $\mu \in \mathbb{R}^{N}$ by $\mu_{i}:=\lambda_{i}^{-1}$ if $\lambda_{i}>0$ and $\mu_{i}:=1$ if $\lambda_{i}=0$. By scale covariance and noting that $L:=\left\{i \in N: x_{i}>\right.$ $0\} \subseteq\left\{i \in N: \lambda_{i}>0\right\}$, we obtain $x=\mu \lambda x \in \Phi\left(\mu V^{\lambda}\right)$. Now the $x_{i}>$ $\mu V^{\lambda}$ is a $\mu^{-1}$-hyperplane game and $V$ satisfies the requirements fone with respect to this hyperplane game as in Property 9.9, controction

\subsection{Applications}

The Shapley transfer procedure and the corresponding results on existence and characterization can be applied to most known solutions for TU-games. Here, we consider applications to the Shapley value, the core, the nucleolus, and the $\tau$-value.

First we state a lemma characterizing the transfer games associated with TU-games. The proof is straightforward and left to the reader. For a vector $\lambda \in \mathbb{R}_{+}^{N} \backslash\{0\}$ and a coalition $S \subseteq N$ denote $\bar{\lambda}_{S}:=\max \left\{\lambda_{i}: i \in\right.$ $S\}$.

Lemma 9.11 Let $v \in \mathcal{T}$ and $\lambda \in \mathbb{R}_{+}^{N} \backslash\{0\}$. Then $v^{\lambda}(S)=\bar{\lambda}_{S} v(S)$ for every coalition $S \subseteq N$. If $x$ is efficient in v, i.e., $\sum_{i \in N} x_{i}=v(N)$, and $\lambda \cdot x=v^{\lambda}(N)$, then $x_{i}=0$ for every $i \in N$ for which $\lambda_{i}<\bar{\lambda}_{N}$

\subsubsection{The Shapley Value}

For a TU-game $v$ the Shapley value $\sigma$ (Shapley, 1953) is defined by $\sigma_{i}(v):=\sum_{S \subseteq N: i \notin S} \frac{|S| !(n-|S|-1) !}{n !}[v(S \cup\{i\})-v(S)]$

for every $i \in N$, where $v(\emptyset):=0$, and $|\cdot|$ denotes the cardinality of a finite set. The Shapley TU-solution assigns the set $\{\sigma(v)\}$ to a game $v$. With some abuse of notation we use $\sigma$ for the Shapley solution and omit the set-brackets. An alternative definition using dividends (cf. Section 3) is also possible. Within our framework the Shapley value is well defined as long as $\sigma(v) \geq 0$. Call an NTU-game $V$ monotonic if $V(S) \subseteq V(T) S$ whene $\sigma(v) \geq T$. Call an NU-game $V$ monotonic if $v(S) \leq v(T)$ whenev $S \subseteq T$. Dente If $U(S) \leq V(T)$ whes of games by $\mathcal{G}_{\text {mon }}$ and $\mathcal{T}_{\text {mon }}$. Then the Shapley value is well defined on $\mathcal{T}_{\text {mon }}$, and $\mathcal{T}_{\text {mon }}=T\left(\mathcal{G}_{\text {mon }}\right)$. It is also straightforward to check that $\sigma$ is a regular TU-solution on $\mathcal{T}_{\text {mon }}$. Moreover, the inclusion in Lemma 9.1 turns out to be an equality, as the following lemma shows.

Lemma 9.12 Let $v \in \mathcal{T}_{\text {mon }}$. Then $\bar{\sigma}(v)=\sigma(v)$.

Proof. Let $\lambda \in \mathbb{R}_{+}^{N} \backslash\{0\}$ and $x \in \mathbb{R}_{+}^{N}$ with $\sum_{i \in N} x_{i}=v(N)$ such that $\lambda x=\sigma\left(v^{\lambda}\right)$. In view of Lemma 9.1 it is sufficient to prove that $x=\sigma(v)$.

\section{$\square \quad 35$}


Let $M:=\left\{i \in N: \lambda_{i}=\bar{\lambda}_{N}\right\}$. Then, by Lemma $9.11, v^{\lambda}(S)=\bar{\lambda}_{N} v(S)$ for every coalition $S$ with $S \cap M \neq \emptyset$, and $x_{i}=0$ for all $i \in N \backslash M$. B monotonicity of $v^{\lambda}$ we have $v^{\lambda}(S) \geq v^{\lambda}(S \cap M)$ for every coalition $S$. By $S \cap M \neq \emptyset$. Suppose $v^{\lambda}(S)>v^{\lambda}(S \cap M)$ for some $S$, then by de with of the Shapley value there must be $i \in N \backslash M$ withen by definition hence $\lambda_{i} x_{i}>0$, a contradiction. coalition $S$ with $S \cap M \neq 0$. Hence $v^{\lambda}(S)=v^{\lambda}(S \cap M)$ for every

We claim that $v(S)=0$ for every coalition $S$ with $S \subseteq N \backslash M$. Suppose not, then there is a $T \subseteq N \backslash M$ with $v(T)>0$. Take $i \in M$ arbitrary. By monotonicity, $v(T \cup\{i\})>0$, hence $v^{\lambda}(T \cup\{i\})=\bar{\lambda}_{N} v(T \cup\{i\})>0$. Therefore, $v^{\lambda}(\{i\})=v^{\lambda}((T \cup\{i\}) \cap M)=v^{\lambda}(T \cup\{i\})>0$, a contradiction since $v(\{i\})=0$ by definition. This proves our claim.

Since $v(S)=0$ whenever $S \cap M=\emptyset$ and $v^{\lambda}(S)=\bar{\lambda}_{N} v(S)$ whenever all $i \in N \backslash M$, we have $v^{\lambda}=\bar{\lambda}_{N} v$. Also, $\lambda x=\bar{\lambda}_{N} x$ since $x_{i}=0$ for $x=\sigma(v)$.

Application of the results in the preceding sections now yields:

Corollary $9.13 \bar{\sigma}(V) \neq \emptyset$ for every $V \in \mathcal{G}_{\text {mon }}$. Moreover, $\bar{\sigma}$ is the unique $N T U$-solution on $\mathcal{G}_{\text {mon }}$ that satisfies Pareto optimality variance, expansion and contraction independence and coincides with the Shapley value on $T_{\text {mon }}$

Proof. Theorem 9.3 implies nonemptiness of $\bar{\sigma}$ on $\mathcal{G}_{\text {mon }}$. The second part follows from Theorem 9.10 and Lemma 9.12.

On the subclass of pure bargaining games $\mathcal{B} \subseteq \mathcal{G}_{\text {mon }}, \tilde{\sigma}$ coincides with the Nash bargaining solution: see the last part of Section 2 .

An earlier characterization of $\bar{\sigma}$ (also called the Shapley NTU-value) was given by Aumann (1985). This characterization presumes exister and makes use of specific properties of the Shapley value; it alo uses the standard concept of unbounded

\subsubsection{The Core}

The core of a TU-game $v$ is defined by

$c(v):=\left\{x \in \mathbb{R}^{N}: \sum_{i \in N} x_{i}=v(N)\right.$

and $\sum_{i \in S} x_{i} \geq v(S)$ for every coalition $\left.S\right\}$.

More generally, the core of an NTU-game $V$ is defined by

$C(V):=\left\{x \in P O(V(N)): \forall \emptyset \neq S \neq N\left[x_{S} \notin \operatorname{int} V(S)\right]\right\}$

where 'int' denotes the topological interior. Nonemptiness of the core is closely connected to the idea of balancedness. A collection of nonnegative numbers $\left\{\gamma_{S}: S\right.$ a coalition $\}$ is called balanced if $\sum_{S: i \in S} \gamma_{S}=1$ for every player $i \in N$. An NTU-game $V$ is called balanced if for every balanced collection $\left\{\gamma_{S}\right\}$ we have $\sum_{S} \gamma_{S} \tilde{V}(S) \subseteq V(N)$, where $V(S) \subset \mathbb{R}^{N}$ is constructed from $V(S)$ by adding zeros for players outside $S$. It is well known (Bondareva, 1963. Shapley, 1967) that a TU-game has a nonempty core if and only if it is balanced. For a NTU-rame, baloncedn cf. Scaf (1967)-inplies nonentiness of the core, but not the cf. Scarf (1967)-implies nonemptiness of the core, but not the other way around.

Let $\mathcal{T}_{\text {bal }}$ denote the class of balanced TU-games, i.e., TU-games with nonempty cores. The TU-solution $c$ is regular, as is easy to verify. Let $\mathcal{G}_{\text {bal }}$ denote the class of balanced NTU-games. By slightly adapting an argument of Qin (1994) ${ }^{1}$ it can be shown that $V \in \mathcal{G}_{\mathrm{bal}}$ if and only if $T(\{V\}) \subseteq \mathcal{T}_{\text {bal }}$. In words, an NTU-game is balanced if and only if all associated transfer games are balanced.

Corollary $9.14 \bar{c}(V) \neq \emptyset$ for every $V \in \mathcal{G}_{\mathrm{bal}}$. Moreover, $\bar{c}$ is the unique $N T U$-solution on $\mathcal{G}_{\text {bal }}$ that satisfies Pareto optimality, scale covariance, expansion and contraction independence and coincides with $\bar{c}$ on $\mathcal{T}_{\mathrm{bal}}$.

Proof. Theorem 9.3 implies nonemptiness of $\bar{c}$ on $\mathcal{G}_{\mathrm{bal}}$. The second part follows from Theorem 9.10.

In this case, applying the transfer procedure on TU-games may add solution outcomes, as the following example shows.

Example 9.15 Consider the four-person TU-game $v$ with player set $N=\{1,2,3,4\}$ and with $v(N)=2, v(S)=1$ if $|S|=3$ or $S \in$ $\{\{1,2\},\{3,4\}\}$, and $v(S)=0$ otherwise. This is a monotonic game with core equal to

$c(v)=\left\{x \in \mathbb{R}_{+}^{N}: x_{1}+x_{2}=1, x_{3}+x_{4}=1\right\}$.

Let $\lambda=(1,1,0,0)$, then $v^{\lambda}$ is equal to $v$ except that $v^{\lambda}(\{3,4\})=0$. Now for $x=(1,1,0,0), \lambda x=(1,1,0,0) \in c\left(v^{\lambda}\right)$, hence $x=(1,1,0,0) \in \bar{c}(v)$ but $x \notin c(v)$.

${ }^{1}$ Attributed to Shapley.

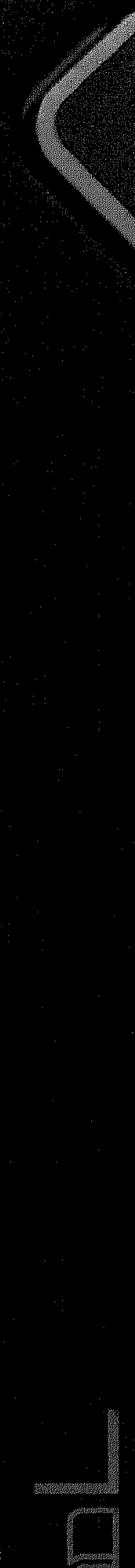


Example 9.15 also implies that the core $C(V)$ of an NTU-game $V$ does not have to contain $\bar{c}(V)$. Also the converse is not true:

Example 9.16 Consider the three-person NTU-game $V$ with player set $N=\{1,2,3\}$ and with $V(N)=\left\{x \in \mathbb{R}_{+}^{N}: x_{1}+x_{2}+x_{3} \leq 3\right\}, V(\{1,2\})=$ $\left\{x \in \mathbb{R}_{+}^{\{1,2\}}: x_{1}+2 x_{2} \leq 3\right\}$, and $V(S)=\{0\}$ otherwise. Note that $(1,1,1) \in C(V)$. The only possible transfer game through which we could obtain $(1,1,1) \in \bar{c}(V)$ would be one correspondinough which wo (or a positive multiple of that vector). For thisonding to $\lambda=(1,1,1)$ $v^{\lambda}(N)=3$ and $v^{\lambda}(\{1,2\})=3$, so thet $\lambda(1,1,1)=(1$ $v^{\lambda}(N)=3$ and $v^{\lambda}(\{1,2\})=3$, so that $\lambda(1,1,1)=(1,1,1) \notin c\left(v^{\lambda}\right)$,
hence $(1,1,1) \notin \bar{c}(V)$.

Example 9.16 still works if we replace the game $V$ by $V^{\prime}$ with as the only difference that now $V^{\prime}(\{1,2\})=\left\{x \in \mathbb{R}_{+}^{\{1,2\}}: x_{1}+3 x_{2} \leq 4\right\}$. In that case, however, the resulting $(1,1,1)$-transfer game has an empty core and therefore $V^{\prime} \notin \mathcal{G}_{\text {bal }}$. The latter fact follows also directly by considerin the collection $\gamma_{\{1,2\}}=1, \gamma_{\{3\}}=1$, and $\gamma_{S}=0$ otherwise. This show that if an NTUnecessarily inherited by a nonempty core, then this property is not necessarily inherited by the associated transfer games. ${ }^{2}$

\subsubsection{The Nucleolus}

The nucleolus (Schmeidler, 1969) for a TU-game $v$ is defined as follows. For every Pareto optimal payoff vector $x$, arrange the $v(S)-\sum_{i \in S} x_{i}(\emptyset \neq S \neq N)$ in a nonincreasing order. $x$ such that the thus associated vector of excen minimal: the resulting payf game has a nonempty The nucleolu $\tau$.

10 apply a 9.10 apply again. Consequently, denoting the nucleolus by $\nu$, we have:

Corollary $9.17 \bar{\nu}(V) \neq \emptyset$ for every $V \in \mathcal{G}$. Moreover, $\bar{\nu}$ is the unique $N T U$-solution on $\mathcal{G}$ that satisfies Pareto optimality, scale covariance, expansion and contraction independence and coincides with $\bar{\nu}$ on $\mathcal{T}$.

Just as was the case with the Shapley value, also the transfer solution as sociated with the nucleolus coincides with the Nash bargaining solution

${ }^{2}$ Qin (1994) also studies an extension of the core to NTU-games by applying the concept of transfer games. These transfer games are $(\lambda-)$ hyperplane games rathe than TU-games, and his approach also differs from ours since in the transfer gam the feasible set of a coalition of players with zero weights is unbounded. In particular, on the subclass of pure bargaining games (see the last part of Section

Like in the case of the core the transfer procedure may add outcomes to TU-games, as is illustrated by the next example.

Example 9.18 Consider the four-person TU-game with $N=\{1,2,3,4\}$ $v(N)=v(\{1,2\})=(\{3,4\})=1$, and $v(S)=0$ otherwise. Then $\nu(v)=\frac{1}{4}(1,1,1,1)$ as is easily derived by symmetry. Take $\lambda=(1,1,0,0)$ then $v^{\lambda}$ is equal to $v$ except that now $v^{\lambda}(\{3,4\})=0$. By symmetry and the fact that the nucleolus is in the core, $\nu\left(v^{\lambda}\right)=\frac{1}{2}(1,1,0,0)$. Hence $\nu\left(v^{\lambda}\right)=\lambda \frac{1}{2}(1,1,0,0)$ so that $\frac{1}{2}(1,1,0,0) \in \bar{\nu}(v)$.

Observe that the game $v$ in this example is not balanced. It is an open question to find an example with a balanced TU-game.

\subsubsection{The $\tau$-Value}

The $\tau$-value for TU-games (Tijs, 1981, Borm et al., 1992) is defined as follows. For a TU-game $v$, define the 'utopia vector' $M(v) \in \mathbb{R}^{N}$ by $M_{i}(v)=v(N)-v(N \backslash\{i\})$ and the 'minimal right vector' $m(v) \in \mathbb{R}^{N}$ by $m_{i}(v)=\max _{S: i \in S}\left(v(S)-\sum_{j \in S \backslash\{i\}} M_{j}(v)\right)$ for every $i \in N$. Then the $\tau$-value $\tau(v)$ is the unique Pareto optimal point on the line segment with $m(v)$ and $M(v)$ as endpoints, if such a point exists and if $m(v) \leq M(v)$. Games for which these two conditions are satisfed ar $m(v) \leq M(v)$. balanced. It can be showo conditions balanced. It can be shown that that every balanced game is quasiWe We will show that transfer games associated with quasi-balanced TU-games are again quasi-balanced. First, we derive some inequalities concerning the utopia and minimal right vectors of transfer games.

Lemma 9.19 Let $v$ be a TU-game and $\lambda \in \mathbb{R}_{+}^{N} \backslash\{0\}$. Then $M_{i}\left(v^{\lambda}\right) \geq \bar{\lambda}_{N} M_{i}(v)$ for all $i \in N$ and

$m_{i}\left(v^{\lambda}\right) \leq \bar{\lambda}_{N} m_{i}(v)$ for all $i \in N$.

Proof. Let $i \in N$. Then, by Lemma 9.11,

$M_{i}\left(v^{\lambda}\right)=v^{\lambda}(N)-v^{\lambda}(N \backslash\{i\})$

$=\bar{\lambda}_{N} v(N)-\bar{\lambda}_{N \backslash\{i\}} v(N \backslash\{i\})$

$\geq \bar{\lambda}_{N}[v(N)-v(N \backslash\{i\})]$

$=\bar{\lambda}_{N} M_{i}(v)$ 
and

$$
\begin{aligned}
m_{i}\left(v^{\lambda}\right) & =\max _{S: S \ni i}\left[v^{\lambda}(S)-\sum_{j \in S \backslash\{i\}} M_{j}\left(v^{\lambda}\right)\right] \\
& \leq \max _{S: S \ni i}\left[\bar{\lambda}_{S} v(S)-\sum_{j \in S \backslash\{i\}} \bar{\lambda}_{N} M_{j}(v)\right] \\
& \leq \max _{S: S \ni i} \bar{\lambda}_{N}\left[v(S)-\sum_{j \in S \backslash\{i\}} M_{j}(v)\right] \\
& =\bar{\lambda}_{N} m_{i}(v) .
\end{aligned}
$$

Here, the before-last inequality follows from the first part of the proos.

Lemma 9.20 Let $v \in \mathcal{T}_{\text {qbal }}$ and $\lambda \in \mathbb{R}_{+}^{N} \backslash\{0\}$. Then $v^{\lambda} \in \mathcal{T}_{\text {qbal }}$. Proof. Let $i \in N$. Then by Lemma 9.19 and the fact that $v \in \mathcal{T}_{\text {qbal }}$ $m_{i}\left(v^{\lambda}\right) \leq \bar{\lambda}_{N} m_{i}(v) \leq \bar{\lambda}_{N} M_{i}(v) \leq M_{i}\left(v^{\lambda}\right)$ and

$\sum_{j \in N} m_{j}\left(v^{\lambda}\right) \leq \bar{\lambda}_{N} \sum_{j \in N} m_{i}(v) \leq \bar{\lambda}_{N} v(N) \leq \bar{\lambda}_{N} \sum_{j \in N} M_{j}(v) \leq \sum_{j \in N} M_{i}\left(v^{\lambda}\right)$, hence $\sum_{j \in N} m_{j}\left(v^{\lambda}\right) \leq v^{\lambda}(N) \leq \sum_{j \in N} M_{i}\left(v^{\lambda}\right)$. So $v^{\lambda} \in \mathcal{T}_{\text {qbal }}$.

We next show that the Shapley transfer procedure does not act outcomes to TU-games. Shapley value. Lemma 9.21 Let $v \in \mathcal{T}_{\text {qbal }}$. Then $\bar{\tau}(v)=\tau(v)$

Proof. Let $\lambda \in \mathbb{R}_{+}^{N} \backslash\{0\}$ and $x \in \mathbb{R}_{+}^{N}$ with $\sum_{i \in N} x_{i}=v(N)$ such that
$\lambda x=\tau\left(v^{\lambda}\right)$. In view

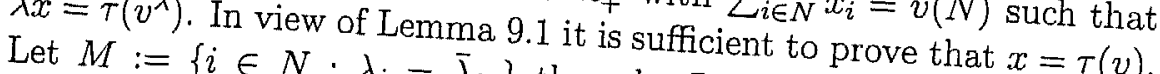
Let $M:=\left\{i \in N: \lambda_{i}=\bar{\lambda}_{N}\right\}$ then, by Lemma $9.11, x_{i}=0$ for all
$i \in N \backslash M$. Hence,

$\tau_{i}\left(v^{\lambda}\right)=0$ for all $i \in N \backslash M$.

Let $\alpha \in[0,1]$ such that $\tau\left(v^{\lambda}\right)=\alpha M\left(v^{\lambda}\right)+(1-\alpha) m\left(v^{\lambda}\right)$.

Case $(a): \alpha=0$

Then

$\tau\left(v^{\lambda}\right)=m\left(v^{\lambda}\right)$, and

$\bar{\lambda}_{N} v(N) \geq \bar{\lambda}_{N} \sum_{j \in N} m_{j}(v) \geq \sum_{j \in N} m_{j}\left(v^{\lambda}\right)=v^{\lambda}(N)=\bar{\lambda}_{N} v(N)$,

where the second inequality follows from Lemma 9.19 , the second equality from Lemma 9.11, and the first equality from (9.2). Hence, all inequalities in (9.3) are equalities. In particular, $m(v)$ is efficient in $v$, so $\tau(v)=m(v)=\left(1 / \bar{\lambda}_{N}\right) \tau\left(v^{\lambda}\right)$. So by (9.1), $x_{i}=0$ and $\tau_{i}(v)=0$ for all $i \in N \backslash M$, so $x_{i}=\tau_{i}(v)$ for these $i$. For $i \in M, x_{i}=\left(1 / \lambda_{i}\right) \tau_{i}\left(v^{\lambda}\right)=$ $\left(1 / \bar{\lambda}_{N}\right) \tau_{i}\left(v^{\lambda}\right)$, hence $x_{i}=\tau_{i}(v)$. Altogether, $x=\tau(v)$.

Case (b): $\alpha>0$

Then for $i \in N \backslash M$, by (9.1), $m_{i}\left(v^{\lambda}\right)=M_{i}\left(v^{\lambda}\right)=0$, hence by Lemm 9.11, $\bar{\lambda}_{N} v(N)=v^{\lambda}(N)=v^{\lambda}(N \backslash\{i\})=\bar{\lambda}_{N} v(N \backslash\{i\})$ so that $M_{i}(v)=0$

$M_{i}(v)=\tau_{i}(v)=0=x_{i}$ for all $i \in N \backslash M$

Now let $i \in M$. First suppose $|M|>1$. Then $M_{i}\left(v^{\lambda}\right)=\bar{\lambda}_{N} v(N)$ $\bar{\lambda}_{N} v(N \backslash\{i\})=\bar{\lambda}_{N} M_{i}(v)$ and

$m_{i}\left(v^{\lambda}\right)=\max _{S: S \ni i}\left[v^{\lambda}(S)-\sum_{j \in S \backslash\{i\}} M_{j}\left(v^{\lambda}\right)\right]=\max _{S: S \ni i}\left[\bar{\lambda}_{N} v(S)-\right.$ $\left.\sum_{j \in S \backslash\{i\}} \lambda_{N} M_{j}(v)\right]=\bar{\lambda}_{N} m_{i}(v)$. Hence $\bar{\lambda}_{N} x_{i}=\tau_{i}\left(v^{\lambda}\right)=\bar{\lambda}_{N} \tau_{i}(v)$, so that $x_{i}=\tau_{i}(v)$. This concludes the proof for $|M|>1$. If $M=\{i\}$ then by (9.4) and efficiency of the $\tau$ value $\tau_{i}(v)=v(N)$ Becouse $\lambda x=\tau\left(v^{\lambda}\right),(9.4)$ and efficiency, we have $\tau_{(}\left(v^{\lambda}\right)=v^{\lambda}(N)=\bar{\lambda}_{N} x_{i}$, hence $x_{i}=v(N)=\tau_{i}(v)$. This concludes the prof of the len $=\lambda_{N} x_{i}$, hence

Denote by $\mathcal{G}_{\text {qbal }}$ the class of NTU-games such that $T\left(\mathcal{G}_{\text {qbal }}\right)=\mathcal{T}_{\text {qbal }}$. By Lemma 9.20 this class contains $\mathcal{T}_{\text {qbal }}$. Moreover, $\mathcal{G}_{\text {qbal }}$ contains the class $\mathcal{G}_{\text {bal }}$ of all balanced NTU-games, since every transfer game associated with a balanced NTU-game is balanced and therefore also quasi-
balanced. be have

We have:

Corollary 9.22 $\bar{\tau}(V) \neq \emptyset$ for every $V \in \mathcal{G}_{\mathrm{abal}}$. Moreover, $\bar{\tau}$ is the unique NTU-solution on $\mathcal{G}_{q}$ that satisfies Pareto optimality, scale covariance, expansion and contraction independence and coincides with the $\tau$-value on $\mathcal{T}_{\text {qbal }}$

Proof. Theorem 9.3 implies nonemptiness of $\bar{\tau}$ on $\mathcal{G}_{\text {qbal }}$. The second part follows from Theorem 9.10 and Lemma 9.21. 
Since on $T(\mathcal{B})$, where as before $\mathcal{B}$ is the class of pure bargaining games, the $\tau$-value coincides with the equal-split solution, it follows again that the transfer solution $\bar{\tau}$ coincides with the Nash bargaining solution on
the class of pure bargaining games.

\subsection{Concluding Remarks}

The main objective of this contribution was to provide existence and Shapley transfor porstutions obtained from TU-solutions by the price paid for this is the same framework price pard for this is the allowance of zero weights and the a the benefit is that the results can be applied to many TU-solutions: see Corollaries 9.13-9.22 The approach followect

existence is less of an issue then we may be modified in many ways. If weights and consider other classes of restrict attention to only positive adopted in the literature. cf. the Harsanyi proce used to extend the proction 3, or the procedure Shapley vale on TU-ganc consistent value-which coincides with 1992)

\section{References}

Aumann, R.J. (1985): "An axiomatization of the non-transferable utility value,", Econometrica, 53, 599-612

Bondareva, O.N. (1963): "Some applications of linear program methods to the theory of coperative games," $P$ 119-139.

Borm, P., H. Keiding, R.P. McLean, S. Oortwijn, and S.H. Tijs (1992): "The Compromise Value for NTU- S. Oortwijn, and S.H. Tijs (1992): Game Theory, 21, 175-189. NTU-Games," International Journal of

Harsanyi, J.C. (1959): "A bargaining model for the cooperative $n$ Press, Princeton, 40,325-355.

cooperative . (1963): "A simplified bargaining model for the $n$-person cooperative game," International Economic Review, 4, 194-220.

Hart, S. (1985): "An axiomatization of Harsanyi's nontransferable utility solution," Econometrica, 53, 1295-1313.

Maschler, M., and G. Owen (1992): "The consistent value for games without side payments," in: R. Selten (ed.), Rational Interaction, 5-12 New York: Springer Verlag.

Nash, J.F. (1950): "The bargaining problem," Econometrica, 18, 155162.

Qin, C.-Z. (1994): "The inner core of an n-person game," Games and Economic Behavior, 6, 431-444

Scarf, H. (1967): "The core of an n-person game," Econometrica, 35 50-67.

Schmeidler, D. (1969): "The nucleolus of a characteristic function game," SIAM Journal of Applied Mathematics, 17, 1163-1170.

Shapley, L.S. (1953): "A value for n-person games," in: H. Kuhn, A.W. Tucker (eds.), Contributions to the Theory of Games, Princeton University Press, Princeton, 307-317.

Shapley, L.S. (1967): "On balanced sets and cores," Naval Research Logistics Quarterly, 14, 453-460.

Shapley, L.S. (1969): "Utility comparison and the theory of games," in G.Th. Guilbaud (ed.), La Décision. Editions du CNRS, Paris.

Shapley, L.S. (1984): "Lecture notes on the inner core," Department of Mathematics, University of California, Los Angeles.

Thomson, W. (1981): "Independence of irrelevant expansions," International Journal of Game Theory, 10, 107-114.

Tijs, S.H. (1981): "Bounds for the core and the $\tau$-value," in: O. Moeschlin and D. Pallasche (eds.), Game Theory and Mathematical Economics, 123-132. Amsterdam: North-Holland. 